\title{
17
}

\section{Complications and Emergencies in the Operating Room}

An emergency can present at any moment in the operating room setting. It is important to be armed with the knowledge and competency to quickly assess and provide treatment to the surgical patient. Some common emergencies in the operating room include motor vehicle accidents resulting in various injuries (e.g., ruptured spleen, cranial bleeding, or broken bones), ruptured aortic aneurysm, and intestinal obstruction.

During this part of your orientation, you will learn about:

- Assessment of the patient during an emergency

- Counting instruments and surgical supplies during an emergency

- Administration of blood products

- The crash/code cart

- Malignant hyperthermia (MH)

- Specialty situations

\section{ASSESSMENT OF THE PATIENT}

It is critical to assess the surgical patient in an emergent situation quickly to determine exactly what will be needed to render the appropriate treatment. 
The basic assessment should include evaluation of:

- Airway

- Breathing

- Circulation

- Pain

- Neurological status

- Dietary status (NPO [nothing by mouth] status)

Vital signs and lab values should be monitored, and any critical or abnormal results should be reported immediately to the anesthesia provider and/or surgeon.

Surgical procedures that are most life-threatening should be performed first, and then the procedures should occur from cleanest (e.g., sterile) to dirtiest (e.g., contaminated).

Additional considerations in emergency situations include:

- Placement of urinary catheter to monitor urine output

- Availability of blood products

- Warming or cooling of patient

- Placement of arterial line or central line

\section{COUNTING INSTRUMENTS AND SURGICAL SUPPLIES}

If a patient is critically ill, the nurse should not take time away from the care of the patient to perform an instrument count. If possible, an attempt to count items (e.g., sponges, sutures, knife blades) should be taken. Also, only x-ray-detectable items should be used on the sterile field.

Regardless of what was counted or uncounted, once the patient is stabilized and surgery is concluded, a portable $\mathrm{x}$-ray should be taken and read by a radiologist to ensure that there is no retained surgical item. Documentation should accurately reflect the emergency and inability to count surgical items.

\section{ADMINISTRATION OF BLOOD PRODUCTS}

Many different types of blood products may be administered in an emergency due to blood and fluid loss. Some common blood products include:

- Packed red blood cells (RBCs)

- Fresh frozen plasma

- Platelets

- Cryoprecipitate 
The administration of blood products requires a crossmatching of a sample of the patient's blood to determine blood type and red cell antibodies.

Administration of blood products usually requires:

- A formal checking process prior to transfusion and a qualified second-person verification

- Use of correct equipment depending on the blood product (e.g., filter, tubing)

- Use of a blood warmer to keep the blood at body temperature

- Transfusion documentation

Complications of administration of blood products must be reported immediately and can include:

- Fever, chills, and urticaria (hives)

- Acute hemolytic transfusion reaction

- Contamination of blood products

\section{Fast Facts}

Question: To administer a unit of RBCs, what are some things you must gather?

Answer: You must gather the blood product, blood tubing, filter if needed, transfusion paperwork, blood warmer, and a qualified person to verify the administration process with you.

\section{CRASH CART FOR SURGICAL EMERGENCIES}

The crash cart, also known as code cart, is strategically located within the operating room semi-restricted area in case of an emergency. It is important to be familiar with the items found on the cart and how to use the defibrillator.

Common items in a crash cart include:

- Emergency medications

- Intravenous (IV) solutions

- 20- to $60-\mathrm{mL}$ syringes

- Central venous catheter

- Arterial blood gas $(\mathrm{ABG})$ collection device

- ECG electrodes

- Cut-down kits for arterial line

- Arterial line kit

- Suction catheters 


\section{Emergency Medications}

Some common emergency medications for adults contained in the crash cart include:

- Amiodarone, 150-mg IV injection

- Atropine, 0.5- to 1-mg IV injection

- Calcium chloride, 0.5- to 1-mg IV injection

- Dopamine, $800-\mathrm{mg}$ vial reconstituted in 500-mL IV bag of D5W (dextrose $5 \%$ in water) or normal saline

- Epinephrine, 1:10,000 1-mg IV injection

- Lidocaine, $100-\mathrm{mg} / 5-\mathrm{mL}$ syringe for injection

- Lidocaine, 2-g vial reconstituted in 500-mL IV bag of D5W or normal saline

- Sodium bicarbonate, $50-\mathrm{mEq} / 50-\mathrm{mL}$ syringe

- Epinephrine injection 1:1,000 $0.3 \mathrm{mg}$

- Narcan 0.4-mg/1-mL to 0.8-mg IV injection

Some of these medications may come in prefilled syringes to make them easier and quicker to administer. All dosages of medications should be checked and confirmed with the providers.

\section{Pediatric Considerations}

Many times, because the dosage of medications and the size of supplies are so different for pediatric patients, a separate and distinct crash cart will be maintained for pediatric patients.

This crash cart can be:

- The same as for adults, but with age- and size-appropriate equipment, supplies, and medications

- A Braselow cart with resuscitation equipment, supplies, and medications

\section{Fast Facts}

A Braselow cart contains a special color-coded system that individualizes equipment and medication dosage relative to the patient's body length. This allows the perioperative team to concentrate on the emergency rather than on drug calculations and sizing an airway.

\section{Defibrillator}

The defibrillator is commonly housed on the top of the crash cart and can be used to electrically shock the heart of the surgical patient during cardiac arrest to help reestablish a normal rhythm. 
A cardiac arrest includes heart rhythms such as:

- Asystole (Figure 17.1)

- Ventricular fibrillation (Figure 17.2)

- Pulseless ventricular tachycardia (Figure 17.3)

- Pulseless electrical activity

Resuscitating (defibrillation) a patient can be accomplished by:

- Applying defibrillator pads to the patient's chest (Figure 17.4)

- Placing external defibrillator paddles on the patient's chest

- Placing internal defibrillator paddles directly on the patient's heart

Table 17.1 describes defibrillator settings.

Cardiopulmonary resuscitation is continued throughout the emergency, if warranted, and medications are given until the patient is stabilized.

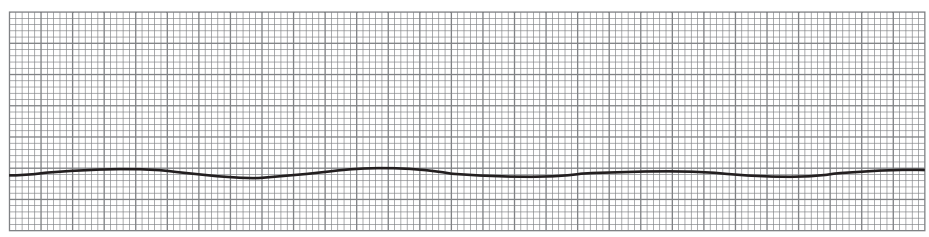

Figure 17.1 Asystole.

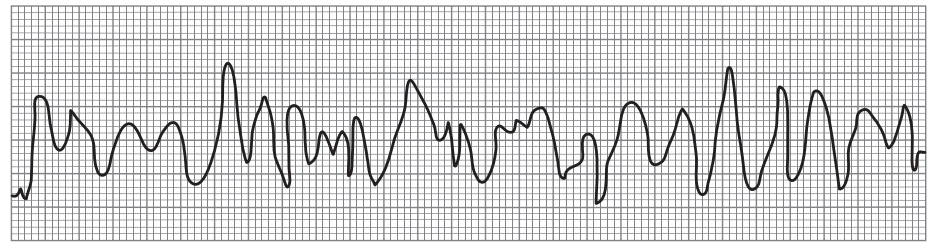

Figure 17.2 Ventricular fibrillation.

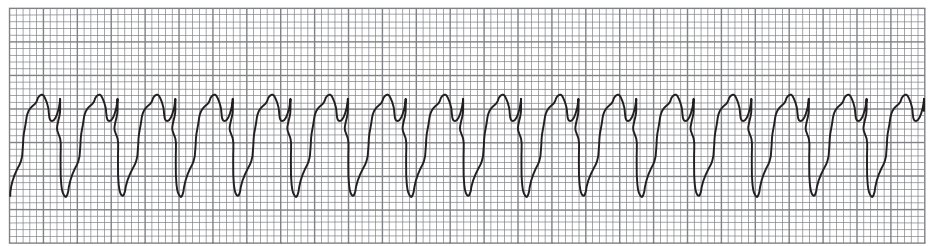

Figure 17.3 Pulseless ventricular tachycardia. 


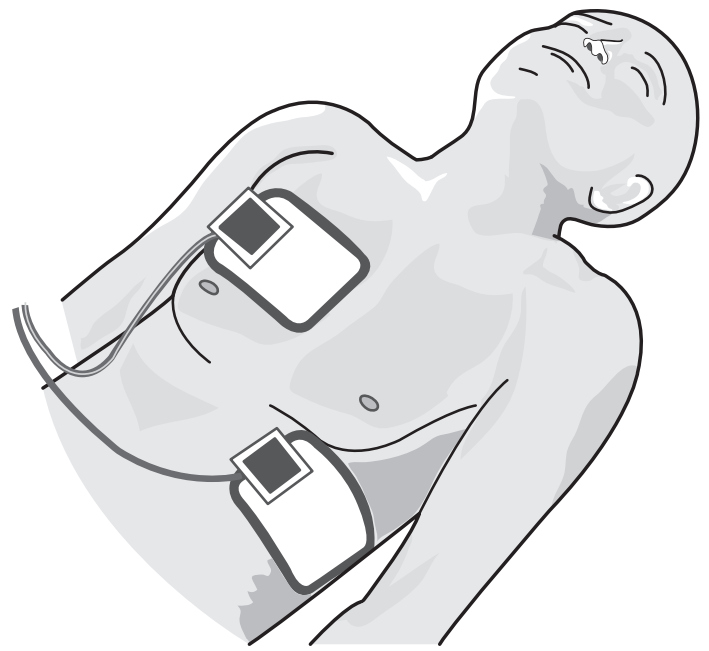

Figure 17.4 Placement of defibrillator pads.

Table 17.1

Defibrillator Settings

\begin{tabular}{lll} 
& External Pad/Paddle & Internal Paddle \\
\hline Adult & $\begin{array}{ll}120-200 \mathrm{~J} \text { (biphasic) } \\
200-360 \text { (monophasic) }\end{array}$ & $<50 \mathrm{~J}$ \\
Child & $\begin{array}{l}2 \mathrm{~J} / \mathrm{kg} \text { of weight (biphasic and } \\
\text { monophasic) }\end{array}$ & $5-50 \mathrm{~J}$ \\
\hline
\end{tabular}

\section{Fast Facts}

The difference between a monophasic and biphasic defibrillator is in how the electrical energy passes through the patient. It is important to keep in mind that with a monophasic defibrillator, the settings are commonly higher.

\section{Additional Testing}

Additional laboratory and other types of testing equipment may be required to evaluate the status of the patient. Some common additional tests may include: 
- ABGs (to check the acidity $[\mathrm{pH}]$ and the levels of oxygen and carbon dioxide in the blood)

- Pericardiocentesis (due to cardiac tamponade, when fluid builds up around the heart due to increased pressure)

- Electrolytes (to determine imbalance and subsequent treatment)

- Cardiac enzymes (to look at enzymes and proteins to determine how much damage has occurred to the heart)

\section{MALIGNANT HYPERTHERMIA}

$\mathrm{MH}$ is a specific concern commonly related directly to the administration of general anesthesia. $\mathrm{MH}$ is a potentially fatal, inherited disorder that is linked to the administration of inhalation anesthetics and/or the muscle relaxant succinylcholine. Therefore, it can manifest as a result of the patient receiving:

- Inhalation anesthesia (e.g., desflurane, enflurane)

- Succinylcholine

This is considered an emergency because, if left undetected and ultimately untreated, it can cause circulatory collapse, and possibly death, in a very short amount of time.

$\mathrm{MH}$ risk factors to consider are:

- It is unusual in the very young and very old.

- Males are affected more than females.

- Patients with trismus (lockjaw) have a higher incidence of positive MH testing.

- Of patients with trismus, $20 \%$ progress to $\mathrm{MH}$ after succinylcholine.

- Patients with some musculoskeletal disorders are at higher risk.

A thorough preoperative screening can help identify potential patients who may exhibit signs and symptoms of $\mathrm{MH}$ or be at risk of $\mathrm{MH}$. Common questions to ask patients include:

- Is there a family history of $\mathrm{MH}$ ?

- Have there been unexpected deaths or complications from anesthesia?

- Is there a personal history of high fever, muscle disorder, or dark-colored urine following anesthesia?

The classic presentation of $\mathrm{MH}$ in the operating room includes:

- Tachycardia (rapid heart rate)

- Rapid rise in end-tidal (ET) $\mathrm{CO}_{2}$ 
- Cardiac arrhythmia

- Acidosis

- Hyperkalemia

- Rigidity

The early signs that should cause the certified surgical technologist (CST), RN, or first assistant concern are:

- Tachycardia and tachypnea (rapid breathing)

- Rapid rise in $\mathrm{ETCO}_{2}$

- Sustained jaw rigidity

- Rapid exhaustion of soda lime that "turns blue"

- Hot soda lime canister

Soda lime is a mixture of chemicals that is used as a filter on the anesthesia machine. This filter removes the $\mathrm{CO}_{2}$ from the breathing circuit. When the patient's $\mathrm{CO}_{2}$ is elevated, the soda lime that is commonly white will turn blue, due to the rapid removal of $\mathrm{CO}_{2}$. The soda lime canister will also feel hot to the touch.

The treatment for $\mathrm{MH}$ includes:

- Discontinue offending agents.

- Hyperventilate with $100 \% \mathrm{O}_{2}$.

- Give dantrolene sodium $2.5 \mathrm{mg} / \mathrm{kg}$ push, repeat as needed (PRN [pro re nata]).

- Treat hyperkalemia (bicarbonate $1-2 \mathrm{mEq} / \mathrm{kg}$ PRN).

- Cool the patient (gastric lavage, ice packs around patient or in wound).

- Measure arterial and/or venous blood gases.

- Obtain electrolytes and coagulation studies.

The Malignant Hyperthermia Association of the United States provides a hotline and detailed information on its website to assist healthcare providers in crisis with patients who have MH. It is important to have this information readily available in each operating room.

1-800-MH-HYPER

$1-800-644-9737$

www.mhaus.org

\section{Fast Facts}

Question: What is the treatment for an MH crisis?

Answer: Discontinue agent, hyperventilate with $100 \% \mathrm{O}_{2}$, give dantrolene sodium $2.5 \mathrm{mg} / \mathrm{kg}$ push IV, and cool the patient using ice packs and/or gastric lavage. 


\section{SPECIALTY SITUATIONS}

Numerous specialty situations can present as an emergency in the operating room. Some such specialty situations are:

- Bullet removal

- Contraband removal

- Multiple-site injuries

- Medical anti-shock trouser (MAST) suit removal

\section{Bullet Removal}

- Bullets should not be handled with metal instruments or placed in metal containers due to the chance of scratching the bullet or compromising the surface.

- Once the bullet is removed and placed in a nonmetal container, it should be submitted to the police as per local and state law enforcement requirements.

- If there is an ongoing criminal investigation, the patient's clothing and belongings may also be secured as evidence. Therefore, the clothing should be cut off the patient prior to surgery along the seams and/or around the bullet- or stab-wound hole. These items should be placed in a paper bag to prevent moisture and growth of mold, which may potentially destroy or compromise the integrity of the evidence.

- Patient statements should also be documented accurately and in detail. Care should be taken to establish the chain of custody of the bullet and any other evidence from point of removal to examination by pathology and law enforcement.

\section{Contraband Removal}

Contraband is considered to be drugs, homemade weapons, and other illegal paraphernalia. These items can come into the operating room hidden in patient body cavities. It is important to:

- Place the contraband in a plastic container, so as to not compromise its integrity.

- Submit contraband to the police as per local and state law enforcement requirements.

- Secure the patient's clothing and belongings as evidence in the event of a criminal investigation.

- Document accurately the patient's statements in detail and establish the chain of custody of the contraband and any other evidence from point of removal to examination by pathology and law enforcement. 


\section{Multiple-Site Injuries}

Multiple-site injuries may require multiple teams of surgeons working on the patient simultaneously. Therefore, these procedures may require multiple scrub RNs/CSTs and multiple circulating nurses. It is important to delegate tasks appropriately to maximize assistance. Also, priority will be given to the most life-threatening injuries. This decision will be made by the anesthesia provider and surgeon.

\section{MAST Garment Removal}

An MAST (medical anti-shock trouser) garment, also known as military anti-shock trouser or pneumatic anti-shock garment, may be used on patients for trauma situations such as hypovolemic shock. This compression garment is placed from the patient's rib line to the ankles and should be inflated if the patient is hypotensive. When removing the MAST garment prior to emergency surgery, it is important to remember that it should not be cut off the patient, but rather deflated while monitoring the blood pressure to prevent a sudden drop in blood pressure or shock.

\section{End-of-Life Care}

At the end of life, care should be taken to consider the cultural and religious beliefs of the individual patient.

Some considerations are:

- Call clergy.

- Have an organ donation representative available to discuss donor opportunities.

- Provide appropriate comfort measures for the patient.

- Provide a designated area for the family to gather privately with the patient.

- Have a palliative care provider available to answer questions.

\section{Fast Facts}

There are three circumstances in which organs can become available for procurement: patient death determined by neurological criteria (brain death), by circulatory or cardiopulmonary criteria, or by voluntary donation, such as a healthy patient donating a kidney. It is becoming more common to allow family in the OR after circulatory 
or cardiopulmonary criteria have been met and the patient is being removed from life support and waiting to die, prior to the procurement of the organs. Because OR staff are commonly advocating for patients and performing life-saving measures, family presence may create anxiety and an uncomfortable feeling for the staff. However, it can help families begin the healing process by knowing that they have created a positive outcome through organ donation (Gysin, Khairallah, \& Reef, 2015).

\section{Reference}

Gysin, D. M., Khairallah, T. S., \& Reef, M. (2015). Donation after circulatory death: Simulating and implementing family presence in the OR. OR Nurse, 9, 29-36. 